\title{
Depressive Symptoms and 24-Hour Ambulatory Blood Pressure in Africans: The SABPA Study
}

\author{
Mark Hamer, ${ }^{1}$ Nancy Frasure-Smith, ${ }^{2,3}$ François Lespérance, ${ }^{2,3}$ Brian H. Harvey, ${ }^{4}$ \\ Nico T. Malan, ${ }^{5}$ and Leoné Malan ${ }^{5}$ \\ ${ }^{1}$ Psychobiology Group, Department of Epidemiology and Public Health, University College London, 1-19 Torrington Place, London \\ WC1E 6BT, UK \\ ${ }^{2}$ Department of Psychiatry and School of Nursing, McGill University CHUM Research Center, QC, Canada \\ ${ }^{3}$ Montreal Heart Institute Research Center, University of Montreal, Montreal, QC, Canada H3C 3J7 \\ ${ }^{4}$ Unit for Drug Research and Development, Division of Pharmacology, School of Pharmacy, North-West University, Potchefstroom 2520, \\ South Africa \\ ${ }^{5}$ Hypertension in Africa Research Team (HART), School for Physiology, Nutrition, and Consumer Sciences, North-West University, \\ Potchefstroom 2520, South Africa
}

Correspondence should be addressed to Mark Hamer, m.hamer@ucl.ac.uk

Received 2 June 2011; Revised 3 August 2011; Accepted 17 August 2011

Academic Editor: Tavis S. Campbell

Copyright (C) 2012 Mark Hamer et al. This is an open access article distributed under the Creative Commons Attribution License, which permits unrestricted use, distribution, and reproduction in any medium, provided the original work is properly cited.

Disturbances in circadian rhythm might play a central role in the neurobiology of depression. We examined the association between depressive symptoms and 24-hour ambulatory BP in a sample of 405 (197 black and 208 Caucasian) urbanized African teachers aged 25 to 60 yrs (mean $44.6 \pm 9.6$ yrs). Depressive symptoms were assessed using the self-administered 9-item Patient Health Questionnaire (PHQ-9). After adjusting for age, sex, and ethnicity, participants with severe depressive symptoms (PHQ-9 $\geq 15$ ) had higher odds of hypertension defined from ambulatory BP and/or use of antihypertensive medication (odds ratio = 2.19, 95\% CI, 1.00-4.90) in comparison to participants with no symptoms. Compared to Caucasians with no depressive symptoms, those with severe symptoms had blunted nocturnal systolic BP drop of $4.7 \mathrm{mmHg}$ (95\% CI, -0.5 to $10.0, P=0.07$ ). In summary, depressive symptoms were associated with the circadian BP profile in black and Caucasian Africans.

\section{Introduction}

Studies examining the association between common mood disorder, such as depression and risk of hypertension, have produced mixed findings. Several studies have reported positive associations between depression and risk of incident hypertension [1-4] whereas others have observed null findings $[5,6]$, or attributed the effects to hypertension labeling [7]. There is even some evidence to suggest lower blood pressure (BP) in participants with depressive symptoms $[8$, 9]. In the Whitehall II study of British civil servants that examined longitudinal trajectories, the risk of hypertension increased with repeated experience of depressive episodes over time and became evident in later adulthood [10]. Thus, the association between depression and hypertension is complex.
One limitation of this body of work is the reliance on clinic BP readings taken on one occasion. In a recent study of older participants that underwent 24-hour ambulatory $\mathrm{BP}$ recordings, those reporting depressive symptoms demonstrated a blunted nocturnal systolic BP fall and were more likely to be nondippers, defined as having nocturnal systolic $\mathrm{BP}$ fall of less than 10\% [11]. There is increasing recognition that disturbances in circadian rhythm play a central role in the neurobiology of depression, even recognizing its importance as a novel pharmacological target in treating the disorder [12]. Depression is associated with alterations in hormone and catecholamine circadian rhythms [13], which may be the result of poorly regulated neurotransmitter feedback control systems that become erratic when chronically stressed. Melatonin, for example, is an endogenous antihypertensive molecule released by the pineal gland that 
plays a role in the biological regulation of circadian rhythms and has been associated with depression. In addition, nocturnal melatonin secretion is impaired among nondipper hypertensive patients [14].

Further studies are required to examine the association between depressive symptoms and the circadian BP profile, which might help to further clarify the equivocal nature of existing literature. The aim of this study was therefore to examine the association between depressive symptoms and circadian BP profiles using 24-hour ambulatory monitoring. The study was conducted in a sample of black and Caucasian Africans who were recruited as part of the Sympathetic Activity and Ambulatory Blood Pressure in Africans (SABPA) study - presently the only study in subSaharan Africa focusing on the contribution of psychosocial risk factors to cardiovascular health. Thus, a further aim was to assess potential ethnic differences in the association between depression and BP.

\section{Methods}

2.1. Participants and Procedures. Participants were recruited as part of the SABPA study conducted between February 2008 and May 2009. The study sample comprised urbanized African teachers working for the Dr. Kenneth Kaunda Education district in the North West Province, South Africa. The reason for this selection was to obtain a homogenous sample from a similar socioeconomic class. All eligible participants between the ages of 25 and 60 years were invited to participate. Exclusion criteria were an oral temperature above $37^{\circ} \mathrm{C}$, psychotropic substance dependence or abuse, blood donors, and individuals vaccinated in the past 3 months. Participants were fully informed about the objectives and procedures of the study prior to their recruitment. Assistance was available for any participant who requested conveyance of information in their home language. All participants signed an informed consent form and the study was approved by The Ethics Review Board of the North-West University (Potchefstroom Campus). Participants were transported at 1630 hours to the Metabolic Unit Research Facility of the North-West University and familiarized with the protocol and completed a battery of psychosocial questionnaires. After receiving a standardized dinner participants were encouraged to go to bed at around 2200 hours and were woken at 0545 hours the following morning to undergo a battery of clinical assessments.

2.2. Depressive Symptoms. Depressive symptoms were assessed using the self-administered 9-item Patient Health Questionnaire (PHQ-9), which has been extensively validated in various clinical patient groups and different ethnic populations including sub-Saharan Africans $[15,16]$. In the present study the PHQ-9 displayed good reliability (Cronbach's alpha $=0.81)$. The PHQ-9 measures the frequency of depressive symptoms corresponding to the 9 key symptoms of depression used by the Diagnostic and Statistical Manual of Mental Disorder Fourth Edition (DSM-IV) criteria to diagnosed major depressive episode.
Participants indicated the frequency of experiencing symptoms during the prior two weeks, ranging from "not at all" (scored as zero), "several days" (one point), "more than half the days" (two points), "nearly every day" (three points), thus giving possible scores of $0-27$. We used previously described PHQ-9 cut points (15) to derive three categories; no depressive symptoms (score $<5$ ), mildmoderate symptoms (score of 5-14), and severe symptoms (score of 15 and above).

2.3. Blood Pressure Measures. Semirecumbent clinic BP was measured twice using a mercury Sphygmomanometer with a 5 -minute rest between each reading. Participants underwent 24-h ambulatory BP monitoring during the working day prior to the clinic visit. At $\sim 0800$ hours, participants were attached with an ambulatory BP monitor (Meditech CE120 CardioTens; Meditech, Budapest, Hungary) on the nondominant arm at their workplace. The apparatus was programmed to measure $\mathrm{BP}$ at $30 \mathrm{~min}$ intervals during the day (0800-2200 hours) and every hour during nighttime (2200-0600 hours). On average, the cuff successfully inflated $72.6 \%$ of the time across all participants. Participants were asked to continue with normal daily activities and record any abnormalities such as headache, nausea, and feeling stressed on their ambulatory diary cards. The data was analyzed using the CardioVisions 1.15 Personal Edition software (Meditech). Hypertension was defined as mean 24hour BP >125/80 mmHg [17], and nondippers were defined as participants having nocturnal systolic BP fall of less than $10 \%$ of their average daytime BP [18].

2.4. Covariates. Sodium fluoride, plasma, and serum samples from fasting blood were stored at $-80^{\circ} \mathrm{C}$ for the analysis of biochemical risk markers. Fasting sodium fluoride (glucose) and serum samples for total and high-density lipoprotein (HDL) cholesterol, gamma glutamyl transferase, and high-sensitivity C-reactive protein (CRP) were analyzed using two sequential multiple analyzers (Konelab 20i; Thermo Scientific, Vantaa, Finland; Unicel DXC 800Beckman and Coulter, Germany). The intra- and intercoefficients of variation for all assays was below $10 \%$.

2.5. Statistical Analyses. Differences in the characteristics of participants in relation to depressive symptoms were analysed using ANOVA tests to examine continuous variables and chi-square tests to examine categorical variables. All CRP values were $\log$ transformed to normalize the distribution. General linear models were used to examine differences in BP between depressive symptoms categories. Logistic regression was used to examine the associations of depressive symptoms with hypertension and nondipper status. Hypertension was defined from BP readings and/or use of antihypertensive medication. The models were adjusted for covariates, including age, sex, ethnicity, CRP, body mass index, total/HDL cholesterol, and glucose. These covariates were selected on an a priori basis, as depressive behavior is accompanied by perturbations in glucose and lipid metabolism, inflammatory, and neural serotonergic system function, all of 
TABLE 1: Characteristics of the study sample $(N=405)$.

\begin{tabular}{|c|c|c|c|c|c|c|}
\hline \multirow[t]{2}{*}{ Variable } & \multicolumn{2}{|c|}{ No depressive symptoms } & \multicolumn{2}{|c|}{ Mild- moderate depressive symptoms } & \multicolumn{2}{|c|}{ Severe depressive symptoms } \\
\hline & Black $(n=42)$ & $\begin{array}{l}\text { Caucasian } \\
(n=101)\end{array}$ & Black $(n=120)$ & $\begin{array}{l}\text { Caucasian } \\
(n=95)\end{array}$ & Black $(n=35)$ & $\begin{array}{c}\text { Caucasian } \\
(n=12)\end{array}$ \\
\hline Age (yrs) & $43.8 \pm 7.3$ & $46.3 \pm 10.8$ & $44.7 \pm 8.3$ & $43.8 \pm 10.7$ & $43.3 \pm 7.8$ & $43.3 \pm 11.3$ \\
\hline Sex (\% men $)$ & 66.7 & 58.4 & 58.8 & 45.7 & $34.3^{*}$ & $33.3^{*}$ \\
\hline Body mass index $\left(\mathrm{kg} \cdot \mathrm{m}^{2}\right)$ & $27.7 \pm 6.6$ & $27.2 \pm 5.4$ & $30.7 \pm 7.2$ & $27.7 \pm 6.5$ & $31.2 \pm 7.1^{*}$ & $29.8 \pm 6.7$ \\
\hline Total/HDL cholesterol ratio & $4.41 \pm 1.85$ & $5.21 \pm 1.63$ & $4.34 \pm 1.58$ & $4.71 \pm 1.58$ & $5.08 \pm 3.31$ & $5.41 \pm 1.69$ \\
\hline C-reactive protein $(\mathrm{mg} / \mathrm{L})^{\dagger}$ & $3.95(54.3)$ & $1.10(14.1)$ & $5.05(53.6)$ & $1.90(26.3)$ & $5.33(32.5)$ & $2.90(25.7)^{*}$ \\
\hline Glucose $(\mathrm{mmol} / \mathrm{L})$ & $5.59 \pm 2.23$ & $5.80 \pm 0.97$ & $5.59 \pm 1.64$ & $5.55 \pm 0.62$ & $5.92 \pm 3.01$ & $5.53 \pm 0.50$ \\
\hline $\begin{array}{l}\text { Antihypertensive medication } \\
\text { usage }(\%)\end{array}$ & 21.5 & 8.9 & 22.5 & 8.4 & 14.3 & 8.3 \\
\hline
\end{tabular}

${ }^{*} P<0.05$ for trend across depressive symptom groups compared within own ethnicity; values displayed as mean \pm SD unless stated $\left({ }^{\dagger}\right.$ non-normally distributed values displayed as median and range).

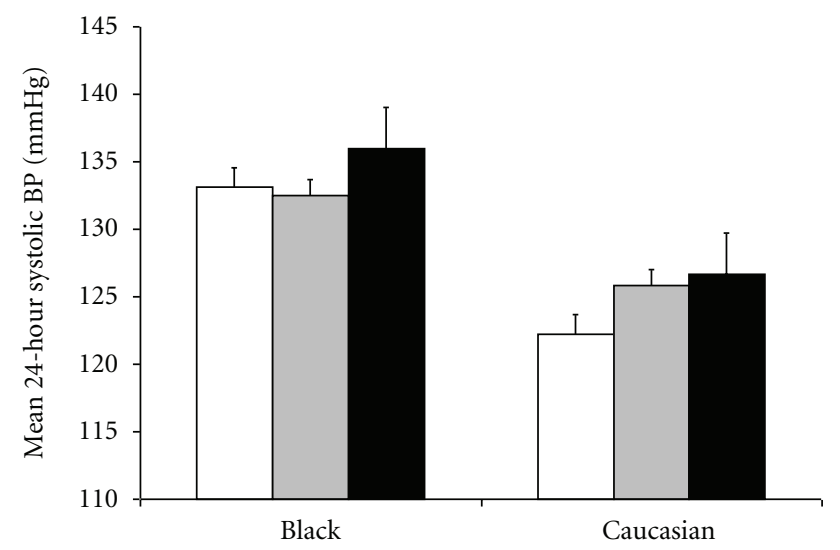

Figure 1: Association between depressive symptoms and mean 24hour ambulatory systolic blood pressure in black and Caucasian Africans. White, grey, and black bars represent none, mildmoderate, and severe depressive symptoms, respectively. Values for blood pressure are mean \pm SEM, adjusted for age, sex, and antihypertensive medication.

which are associated with exacerbated hypertension [19]. We fitted interaction terms in order to assess potential ethnic differences in the association between depression and BP. All analyses were conducted using SPSS version 14.

\section{Results}

The sample comprised 197 black (aged $44.2 \pm 8.0 \mathrm{yrs)}$ ) and 208 Caucasians (aged $45.0 \pm 10.8$ yrs). Severe depressive symptoms were reported in $11.6 \%$ of the sample. Black participants were more likely to report any depressive symptoms compared with Caucasians (age- and sex-adjusted odds ratio $=3.72,95 \%$ CI, 2.35-5.89). Depressive symptoms were associated with several risk factors including higher body mass index and elevated CRP (see Table 1). In addition, black ethnicity was strongly related to other risk factors, including elevated CRP, BMI, and BP.

Hypertension was prevalent in $62.1 \%$ of the sample when using the 24-hour ambulatory BP criteria. There was a strong
TABLE 2: The association between depressive symptoms and hypertension defined from $24 \mathrm{hr}$ ambulatory blood pressure readings. $(N=405)$.

\begin{tabular}{lccc}
\hline $\begin{array}{l}\text { Depressive } \\
\text { symptoms }\end{array}$ & Cases/N & $\begin{array}{c}\text { Model 1 OR } \\
(95 \% \mathrm{CI})\end{array}$ & $\begin{array}{c}\text { Model 2 OR } \\
(95 \% \mathrm{CI})\end{array}$ \\
\hline None & $80 / 143$ & Reference & Reference \\
Mild-moderate & $142 / 215$ & $1.91(1.15-3.18)$ & $1.79(1.02-3.13)$ \\
Severe & $32 / 47$ & $2.19(1.00-4.90)$ & $1.86(0.76-4.57)$ \\
\hline
\end{tabular}

${ }^{\ddagger}$ defined as mean $24 \mathrm{hr} \mathrm{BP} \geq 125 / 80 \mathrm{mmHg}$ and/or use of antihypertensive medication.

Model 1; adjustment for age, sex, ethnicity.

Model 2; adjustment for age, sex, ethnicity, + C-reactive protein, body mass index, total/HDL cholesterol, glucose.

correlation between clinic and ambulatory systolic BP $(r=$ $0.74, P<0.001)$. Black participants recorded higher 24 -hour systolic BP (age-, sex-, medication-adjusted $\beta=7.8,95 \%$ CI, $5.0-10.6 \mathrm{mmHg}$ ) compared to Caucasians. There was an association between depressive symptoms and 24-hour ambulatory BP (see Figure 1). For example, compared to participants with no depressive symptoms, those with severe symptoms had marginally increased 24-hour systolic BP of $4.2 \mathrm{mmHg}(95 \% \mathrm{CI},-0.5$ to $8.9, P=0.08)$ after adjustment for age, sex, ethnicity, and antihypertensive medication. Depressive symptoms were associated with a twofold risk of hypertension defined from 24-hour ambulatory BP monitoring (Table 2), although when hypertension was defined from clinic BP readings $(\geq 140 / 90 \mathrm{mmHg})$ there was no associations with depression (odds ratio of hypertension for severe depressive symptoms $=1.36,95 \%$ CI, $0.63-$ 2.96). The associations of depressive symptoms and 24hour hypertension were slightly attenuated after further adjustments for other risk factors including body mass index, CRP, total/HDL cholesterol, and glucose. We found no evidence for any statistically significant ethnic differences in the association between depression and BP. The removal of four participants who reported use of antidepressant medication did not alter any of the results.

Fifty-nine participants (14.5\%) recorded experiencing stress on their diary cards during the ambulatory monitoring 
TABLE 3: The association between depressive symptoms and nondipper status.

\begin{tabular}{lccc}
\hline $\begin{array}{l}\text { Depressive } \\
\text { symptoms }\end{array}$ & Cases/N & $\begin{array}{c}\text { Model 1 OR } \\
(95 \% \mathrm{CI})\end{array}$ & $\begin{array}{c}\text { Model 2 OR } \\
(95 \% \mathrm{CI})\end{array}$ \\
\hline None & $45 / 143$ & Reference & Reference \\
Mild-moderate & $75 / 215$ & $1.04(0.65-1.67)$ & $1.01(0.62-1.63)$ \\
Severe & $23 / 47$ & $1.74(0.85-3.58)$ & $1.74(0.83-3.63)$ \\
\hline
\end{tabular}

Model 1; adjustment for age, sex, ethnicity, antihypertensive medication. Model 2; adjustment for age, sex, ethnicity, antihypertensive medication + C-reactive protein, BMI, total/HDL cholesterol, glucose.

day, and those participants had elevated 24-hour systolic BP compared with nonstressed (age-, sex-, ethnicity-adjusted $\beta=5.5,95 \% \mathrm{CI}, 1.7$ to $9.3 \mathrm{mmHg}, P=0.004$ ). Participants reporting severe depressive symptoms were more likely to report feeling stressed (age-, sex-, ethnicity-adjusted odds ratio $=2.96,95 \% \mathrm{CI}, 1.16-7.50)$. When we added stress as a covariate, the difference in 24-hour systolic BP between participants reporting none and severe depressive symptoms was no longer evident ( $\beta=3.3,95 \% \mathrm{CI},-1.4$ to $8.0 \mathrm{mmHg}$, $P=0.16$ ), which suggests stress might partly explain the link between depression and the circadian BP profile.

We further examined the association between depressive symptoms and nocturnal BP decline (Table 3 and Figure 2). A nocturnal systolic BP fall of less than 10\% (nondipper) was observed in $31.4 \%$ of the sample, and black participants were more likely to be nondippers compared with Caucasians (age-, sex-, medication-adjusted odds ratio $=1.62,95 \% \mathrm{CI}$, 1.04-2.51). There was a weak association between depressive symptoms and nocturnal BP decline that was particularly evident in Caucasians. For example, compared to Caucasians with no depressive symptoms, those with severe symptoms had blunted nocturnal systolic BP drop of $4.7 \mathrm{mmHg}$ ( $95 \%$ $\mathrm{CI},-0.5$ to $10.0, P=0.07$ ) after adjustment for age, sex, and antihypertensive medication (Figure 2). In the whole sample there was evidence of elevated risk of nondipping in participants with severe depressive symptoms although this did not reach statistical significance (Table 3), nor was there any significant interaction by ethnicity.

\section{Discussion}

This is presently the only study in sub-Saharan Africa to examine the association between depressive symptoms and risk of hypertension. Black Africans are known to have elevated BP compared with Caucasians [20], and this was confirmed in the present study. Black participants from the present study also demonstrated a higher prevalence of depressive symptoms. Despite this, the association between depressive symptoms and BP remained independent of ethnicity and there was no evidence for any significant ethnic differences in the association between depression and BP.

Previous studies in this area have been limited by only taking clinic BP readings, but in the present study we also collected data from 24-hour ambulatory BP monitoring. Our main findings show an association between depressive symptoms and 24-hour ambulatory BP. To our knowledge,

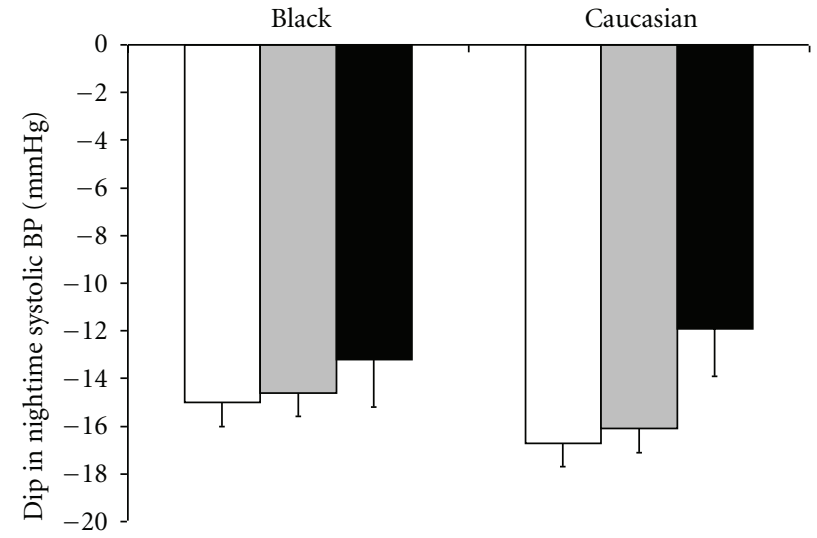

Figure 2: Association between depressive symptoms and change in ambulatory systolic blood pressure between daytime and night in black and Caucasian Africans. White, grey, and black bars represent none, mild-moderate, and severe depressive symptoms, respectively. Values for blood pressure are mean \pm SEM, adjusted for age, sex, and antihypertensive medication.

only one other study examining depression has employed ambulatory BP monitoring [11]. They found an association between depressive symptoms and blunted nocturnal systolic BP fall. Our findings are partly consistent with those results although we did not find strong evidence for an association between depression and risk of nondipping, which might be explained by the older age of participants from the previous study. Several large epidemiological cohort studies have found evidence for an association between depression and elevated risk of incident hypertension at followup [1$3,10]$. However, in other studies depressive disorder was associated with lower systolic BP $[8,9]$, although use of tricyclic antidepressants was associated with greater risk of hypertension $[5,8]$. These inconsistencies might therefore be partially explained by inadequate assessments of BP, antidepressant medication, different measures of symptoms and diagnostic criteria for depression, or other confounding factors.

The associations between depressive symptoms and 24hour BP were attenuated after further adjustments for other risk factors including body mass index, CRP, cholesterol, and glucose. However, given that these factors might act as intermediate mechanisms on the causal pathway from depression to hypertension, our models may have been overadjusted. For example, depressive symptoms were associated with body mass index and CRP, both of which have been implicated in hypertension risk [21,22]. Indeed, these mechanisms have also been linked with the association between depression and coronary heart disease [23-25].

From our results we might speculate that daily stress partly explains the link between depression and the circadian BP profile. Participants with severe depressive symptoms were more likely to report feeling stressed on the ambulatory BP monitoring day, which corroborates with observations that depressed individuals are more likely to report events as stressful and to complain and endorse somatic symptoms. 
Heightened BP reactivity to laboratory-induced stressors has been prospectively associated with a greater risk of hypertension [26], thus if elicited repeatedly in a person's life these responses might be clinically relevant. Moreover, there is also overlapping biology between the neurohormonal control of $\mathrm{BP}$ and the subsequent downstream effects of psychological stress, one in particular being the gaseous transmitter and neuroregulator, nitric oxide [27]. Indeed, naturalistic studies have shown profound effects of acute stressors on BP. For example, the World Trade Centre terrorist attacks in New York on 11th September 2001 produced a substantial and sustained increase in the BP of participants from the local community [28]. There was also a $20 \%$ increase in systolic BP following a moderate intensity earth quake that struck central Italy, which was followed by a long lasting period of enhanced BP variability and blunted nocturnal BP fall [29]. Work stress has also been associated with elevated 24-hour ambulatory BP [30] although the findings from prospective cohort studies in relation to work stress and hypertension risk have been generally inconsistent [31-36].

The strengths of this study include the unique sample of participants from sub-Saharan Africa, a well-validated measure of depressive symptoms, and the use of 24-hour ambulatory BP assessments. A limitation is the crosssectional design, thus we cannot infer causality, identify potential mediators, nor determine the direction of the observed relationship between depression and hypertension. In addition, we did not assess history of major depression and recurrent symptoms, which might be important given the link between persistent depressive symptoms and cardiovascular risk [37]. In summary, we found an association between depressive symptoms and 24-hour ambulatory BP in black and Caucasian Africans. The equivocal nature of the existing work in this area might be explained by reliance on clinic BP readings and failure to capture the circadian BP profile.

\section{Funding}

Funding was provided by HART (North-West University) and National Research Foundation South Africa, Metabolic Syndrome Institute, France. Dr Hamer is supported by the British Heart Foundation, UK. The funding organizations played no role in the design and conduct of the study; collection, management, analysis, and interpretation of the data; preparation, review, or approval of the paper.

\section{Author's Contributions}

M. Hamer had full access to the data and takes responsibility for the integrity of the data and accuracy of the data analyses. M. Hamer performed statistical analyses. L. Malan is the principle investigator and led the study. All authors contributed to the concept and design of study, drafting, and critical revision of the paper.

\section{Conflict of Interests}

The authors declare that there is no conflict of interest.

\section{Acknowledgments}

The authors thank Chrissie Lessing (research nurse) and Szabolcs Péter (MD) for their contribution to data collection and analysis.

\section{References}

[1] S. B. Patten, J. V. A. Williams, D. H. Lavorato, N. R. C. Campbell, M. Eliasziw, and T. S. Campbell, "Major depression as a risk factor for high blood pressure: epidemiologic evidence from a national longitudinal study," Psychosomatic Medicine, vol. 71, no. 3, pp. 273-279, 2009.

[2] B. S. Jonas, P. Franks, and D. D. Ingram, "Are symptoms of anxiety and depression risk factors for hypertension? Longitudinal evidence from the National Health and Nutrition Examination Survey I Epidemiologic Follow-up Study," Archives of Family Medicine, vol. 6, no. 1, pp. 43-49, 1997.

[3] J. H. Markovitz, K. A. Matthews, W. B. Kannel, J. L. Cobb, and R. B. D’Agostino, "Psychological predictors of hypertension in the Framingham study. Is there tension in hypertension?" Journal of the American Medical Association, vol. 270, no. 20, pp. 2439-2443, 1993.

[4] D. Carroll, A. C. Phillips, C. R. Gale, and G. D. Batty, "Generalized anxiety and major depressive disorders, their comorbidity and hypertension in middle-aged men," Psychosomatic Medicine, vol. 72, no. 1, pp. 16-19, 2010.

[5] J. A. C. Delaney, B. E. Oddson, H. Kramer, S. Shea, B. M. Psaty, and R. L. McClelland, "Baseline depressive symptoms are not associated with clinically important levels of incident hypertension during two years of follow-up: the multi-ethnic study of atherosclerosis," Hypertension, vol. 55, no. 2, pp. 408$414,2010$.

[6] L. L. Yan, K. Liu, K. A. Matthews, M. L. Daviglus, T. F. Ferguson, and C. I. Kiefe, "Psychosocial factors and risk of hypertension: the Coronary Artery Risk Development in Young Adults (CARDIA) study," Journal of the American Medical Association, vol. 290, no. 16, pp. 2138-2148, 2003.

[7] M. Hamer, G. D. Batty, E. Stamatakis, and M. Kivimaki, "Hypertension awareness and psychological distress," Hypertension, vol. 56, no. 3, pp. 547-550, 2010.

[8] C. M. Licht, E. J. de Geus, A. Seldenrijk et al., "Depression is associated with decreased blood pressure, but antidepressant use increases the risk for hypertension," Hypertension, vol. 53, no. 4, pp. 631-638, 2009.

[9] B. Hildrum, A. Mykletun, E. Stordal, I. Bjelland, A. A. Dahl, and J. Holmen, "Association of low blood pressure with anxiety and depression: the Nord-Trøndelag Health Study," Journal of Epidemiology and Community Health, vol. 61, no. 1, pp. 53-58, 2007.

[10] H. Nabi, J. F. Chastang, T. Lefèvre et al., "Trajectories of depressive episodes and hypertension over 24 years: the whitehall II prospective cohort study," Hypertension, vol. 57, pp. 710-716, 2011.

[11] A. Scuteri, G. Spalletta, M. Cangelosi et al., "Decreased nocturnal systolic blood pressure fall in older subjects with depression," Aging-Clinical and Experimental Research, vol. 21, no. 4-5, pp. 292-297, 2009.

[12] C. R. Dolder, M. Nelson, and M. Snider, "Agomelatine treatment of major depressive disorder," Annals of Pharmacotherapy, vol. 42, no. 12, pp. 1822-1831, 2008.

[13] H. W. Koenigsberg, M. H. Teicher, V. Mitropoulou et al., "24-h Monitoring of plasma norepinephrine, MHPG, cortisol, 
growth hormone and prolactin in depression," Journal of Psychiatric Research, vol. 38, no. 5, pp. 503-511, 2004.

[14] M. Jonas, D. Garfinkel, N. Zisapel, M. Laudon, and E. Grossman, "Impaired nocturnal melatonin secretion in nondipper hypertensive patients," Blood Pressure, vol. 12, no. 1, pp. 19-24, 2003.

[15] K. Kroenke, R. L. Spitzer, and J. B. W. Williams, "The PHQ9: validity of a brief depression severity measure," Journal of General Internal Medicine, vol. 16, no. 9, pp. 606-613, 2001.

[16] P. O. Monahan, E. Shacham, M. Reece et al., "Validity/reliability of PHQ-9 and PHQ-2 depression scales among adults living with HIV/AIDS in western Kenya," Journal of General Internal Medicine, vol. 24, no. 2, pp. 189-197, 2009.

[17] G. Mancia, G. De Backer, A. Dominiczak et al., "Guidelines for the management of arterial hypertension: the Task Force for the Management of Arterial Hypertension of the European Society of Hypertension (ESH) and of the European Society of Cardiology (ESC)," Journal of Hypertension, vol. 25, no. 6, pp. 1105-1187, 2007.

[18] P. Verdecchia, G. Schillaci, M. Guerrieri et al., "Circadian blood pressure changes and left ventricular hypertrophy in essential hypertension," Circulation, vol. 81, no. 2, pp. 528536, 1990.

[19] C. A. Shively, D. L. Musselman, and S. L. Willard, "Stress, depression, and coronary artery disease: modeling comorbidity in female primates," Neuroscience and Biobehavioral Reviews, vol. 33, no. 2, pp. 133-144, 2009.

[20] G. Dwivedi and D. G. Beevers, "Hypertension in ethnic groups: epidemiological and clinical perspectives," Expert Review of Cardiovascular Therapy, vol. 7, no. 8, pp. 955-963, 2009.

[21] K. Rahmouni, M. L. G. Correia, W. G. Haynes, and A. L. Mark, "Obesity-associated hypertension: new insights into mechanisms," Hypertension, vol. 45, no. 1, pp. 9-14, 2005.

[22] P. Mathieu, P. Poirier, P. Pibarot, I. Lemieux, and J. P. Després, "Visceral obesity: the link among inflammation, hypertension, and cardiovascular disease," Hypertension, vol. 53, no. 4, pp. 577-584, 2009.

[23] V. Vaccarino, B. D. Johnson, D. S. Sheps et al., "Depression, inflammation, and incident cardiovascular disease in women with suspected coronary ischemia: the National Heart, Lung, and Blood Institute-sponsored WISE study," Journal of the American College of Cardiology, vol. 50, no. 21, pp. 2044-2050, 2007.

[24] V. Vaccarino, C. McClure, B. D. Johnson et al., "Depression, the metabolic syndrome and cardiovascular risk," Psychosomatic Medicine, vol. 70, no. 1, pp. 40-48, 2008.

[25] W. J. Kop, P. K. Stein, R. P. Tracy, J. I. Barzilay, R. Schulz, and J. S. Gottdiener, "Autonomic nervous system dysfunction and inflammation contribute to the increased cardiovascular mortality risk associated with depression," Psychosomatic Medicine, vol. 72, no. 7, pp. 626-635, 2010.

[26] Y. Chida and A. Steptoe, "Greater cardiovascular responses to laboratory mental stress are associated with poor subsequent cardiovascular risk status: a meta-analysis of prospective evidence," Hypertension, vol. 55, no. 4, pp. 1026-1032, 2010.

[27] F. Oosthuizen, G. Wegener, and B. H. Harvey, "Nitric oxide as inflammatory mediator in post-traumatic stress disorder (PTSD): evidence from an animal model," Journal of Neuropsychiatric Disease and Treatment, vol. 1, pp. 109-123, 2005.

[28] W. Gerin, W. Chaplin, J. E. Schwartz et al., "Sustained blood pressure increase after an acute stressor: the effects of the 11 September 2001 attack on the New York City World Trade
Center," Journal of Hypertension, vol. 23, no. 2, pp. 279-284, 2005.

[29] G. Parati, R. Antonicelli, F. Guazzarotti, E. Paciaroni, and G. Mancia, "Cardiovascular effects of an earthquake: direct evidence by ambulatory blood pressure monitoring," Hypertension, vol. 38, no. 5, pp. 1093-1095, 2001.

[30] T. G. M. Vrijkotte, L. J. P. van Doornen, and E. J. C. De Geus, "Effects of work stress on ambulatory blood pressure, heart rate, and heart rate variability," Hypertension, vol. 35 , no. 4, pp. 880-886, 2000.

[31] J. H. Markovitz, K. A. Matthews, M. Whooley, C. E. Lewis, and K. J. Greenlund, "Increases in job strain are associated with incident hypertension in the CARDIA Study," Annals of Behavioral Medicine, vol. 28, no. 1, pp. 4-9, 2004.

[32] A. Chapman, J. A. Mandryk, M. S. Frommer, B. V. Edye, and D. A. Ferguson, "Chronic perceived work stress and blood pressure among Australian government employees," Scandinavian Journal of Work, Environment and Health, vol. 16, no. 4, pp. 258-269, 1990.

[33] C. Guimont, C. Brisson, G. R. Dagenais et al., "Effects of job strain on blood pressure: a prospective study of male and female white-collar workers," American Journal of Public Health, vol. 96, no. 8, pp. 1436-1443, 2006.

[34] P. L. Schnall, J. E. Schwartz, P. A. Landsbergis, K. Warren, and T. G. Pickering, "A longitudinal study of job strain and ambulatory blood pressure: results from a three-year followup," Psychosomatic Medicine, vol. 60, no. 6, pp. 697-706, 1998.

[35] J. P. Fauvel, I. M'Pio, P. Quelin, J. P. Rigaud, M. Laville, and M. Ducher, "Neither perceived job stress nor individual cardiovascular reactivity predict high blood pressure," Hypertension, vol. 42, no. 6, pp. 1112-1116, 2003.

[36] B. Öhlin, G. Berglund, M. Rosvall, and P. M. Nilsson, "Job strain in men, but not in women, predicts a significant rise in blood pressure after 6.5 years of follow-up," Journal of Hypertension, vol. 25, no. 3, pp. 525-531, 2007.

[37] M. Hamer, M. Kivimaki, A. Lahiri, M. G. Marmot, and A. Steptoe, "Persistent cognitive depressive symptoms are associated with coronary artery calcification," Atherosclerosis, vol. 210, no. 1, pp. 209-213, 2010. 


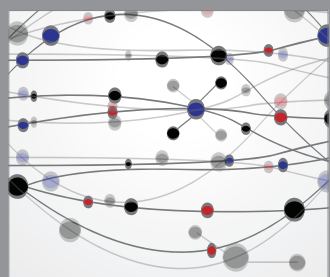

The Scientific World Journal
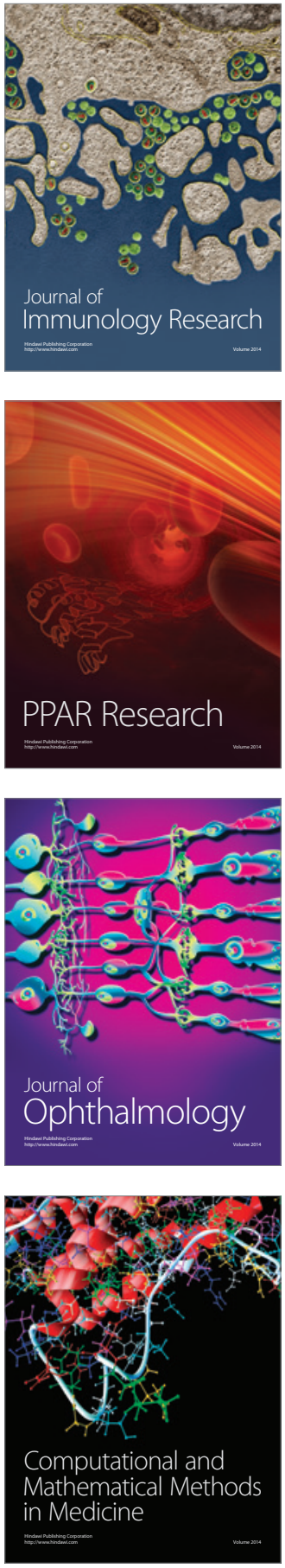

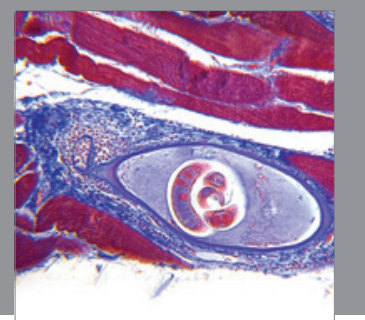

Gastroenterology

Research and Practice
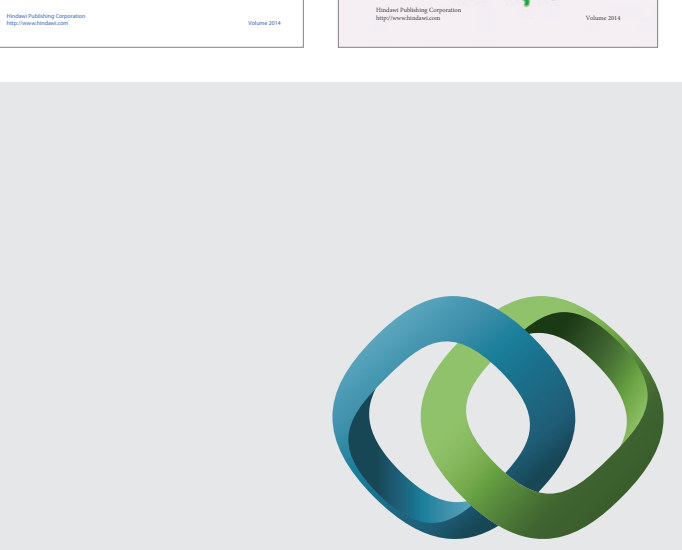

\section{Hindawi}

Submit your manuscripts at

http://www.hindawi.com
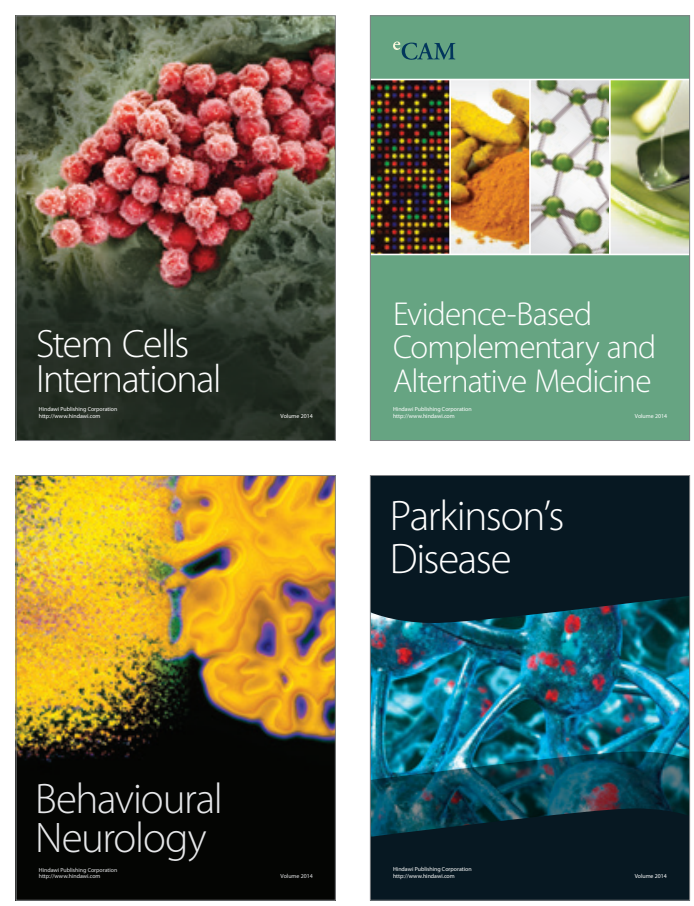

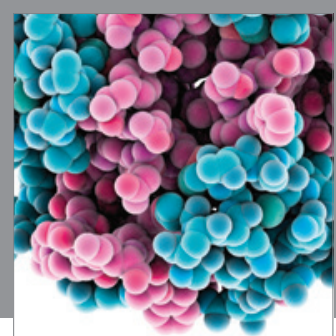

Journal of
Diabetes Research

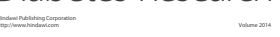

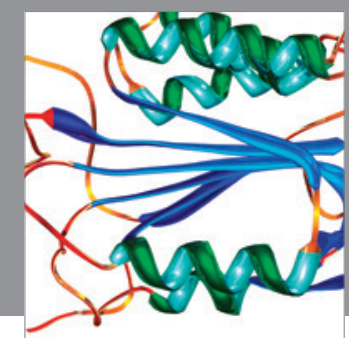

Disease Markers
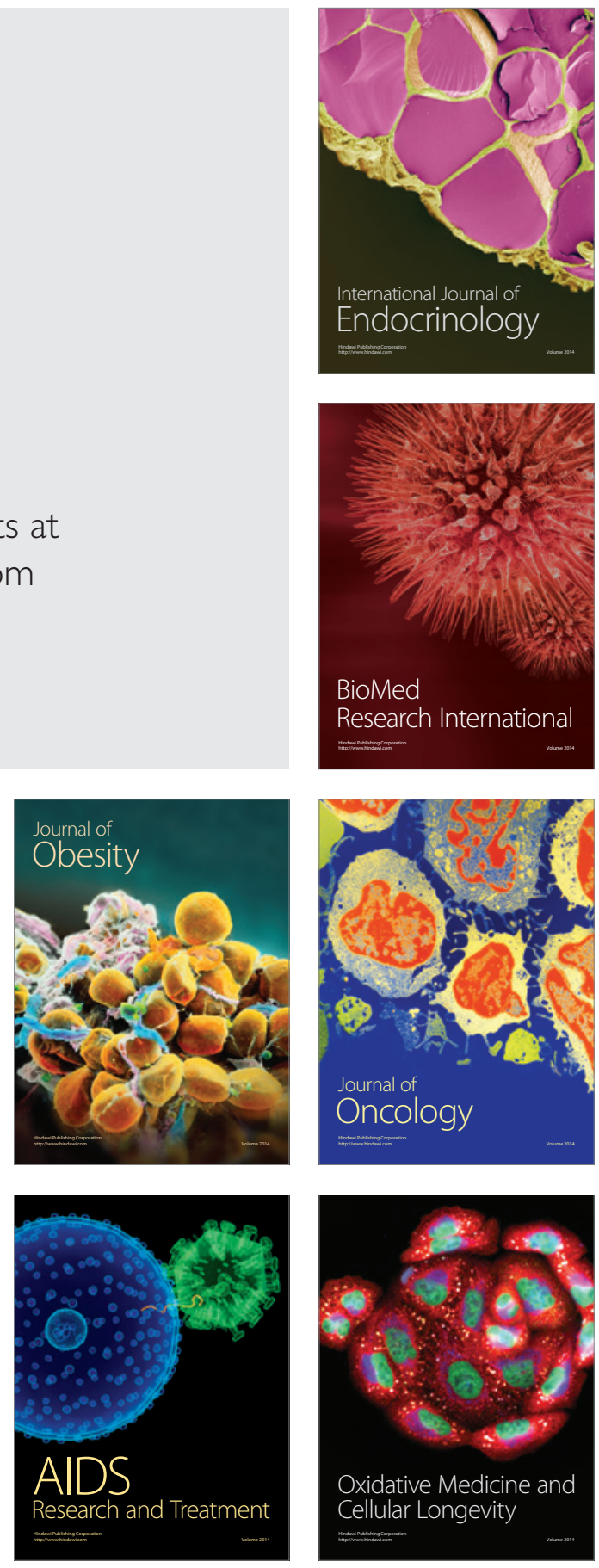